\title{
OBSERVATIONS ON SPEECH DISORDERS IN CHILDREN
}

By C. Worster-Drought, M.A., M.D., F.R.C.P.

Consulting Physician to the West End Hospital for Neurology, and formerly in charge of the Department of Speect Disorders; Consulting Physician-Neurologist, Metropolitan Hospital, London; Hon. Medical Director, Moor House School for Children Suffering from Speech Disorders, Oxted, Snrrey

Speech is one of the two principal methods by means of which we communicate with our fellowmen, the second method being the use of written or printed symbols. To 'speak' is not merely to utter words, the faculty of 'speaking' being essentially a mental process, involving the construction of propositions. Each country has its own particular code or language which is learned in childhood by its inhabitants, in the first instance by sound. The normal child, at first, does not recognize words as such, but distinguishes their implication from the intonation, pitch and rhythm of the speaker's voice in the same way as the more intelligent animals, such as the dog and horse. Later, the child learns to attach meaning to individual words and, still later, by the laborious process of education, a knowledge of the conventional symbols of written or printed words is acquired; thus spoken words can be permanently recorded. Words, by association, eventually stand for the objects or actions to which they refer. When the child reacts in the same way to a word as he would to the situation for which the word stands, he is said to know the 'meaning' of the word.

By a 'speech disorder' we mean some defect in the faculty of speaking. It is necessary to distinguish between ( $I$ ) the primary factor of speech, which is a function of the brain, and (2) articulation, which depends on the integrity of the nervous and muscular structures concerned with the utterance of words. These structures include the various nerve cells and fibres in the brain and in the medulla or bulb, the peripheral nerves to which the latter give rise, and the muscular structures of the lips, tongue, soft palate, larynx and pharynx which these nerves supply. A disturbance of the cortical or higher speech areas, or of their connections in the brain, results in the disorder of speech known as 'aphasia'. When the speech defect is due to imperfect articulation, the term 'dysarthria' is employed. Thus, we recognise two main classes of speech disorder :
(I) Aphasia and (2) Dysarthria.

In patients suffering from aphasia, the highe intellectual processes continue to function although in some cases imperfectly, but the cortical areas which control either the perceptive process of recognizing spoken language or the motor act of speaking are disordered ofo undeveloped, yet the patient has no paralysis of the muscles concerned with speech nor is he deaf or blind. In other words, 'aphasia' involves ob disorder in the comprehension and/or the for $\frac{\text { o }}{0}$ mulation of words and sentences which probably depends on a deficiency of memory for those symbols, whether spoken or written, by meansofofo which we exchange ideas with other individuass: An aphasic patient, unless his mental faculties impaired, can usually communicate quite well means of gesture and pantomime.

For a perfect interchange of ideas two maing processes may be postulated: (I) Reception and (2) Execution or Expression. The receptive process includes the hearing and comprehension of spoker? language; also the seeing and understanding of written or printed words and figures. The memories of words heard and seen appear to depend on the integrity of certain specialized areas of the auditory and visual portions of the cerebral cortex termed respectively the 'auditory word area 3 . and the 'visual word area'. The former is situated. in the superior temporal convolution of the brain and the visual word area in the region of theo angular gyrus. Either of these areas may be relatively undeveloped, injured or diseased. Con sequently, we can distinguish two varieties of disordered reception or 'receptive aphasia' $O$ (I) Auditory aphasia - the inability to understand spoken language although hearing itself is intact ${ }_{\mathrm{W}}$ also known as ' word-deafness', and (2) Visuab aphasia-the inability to appreciate written of printed language although sight is normal, alsoक termed ' word blindness', alexia or dyslexia. \&

The expressive or executive element in speecho consists of the motor act of self-expression in 
words, either vocally by talking or by means of writing. Every voluntary motor act learned by an individual is accompanied by a conscious sensation of activity, and by repetition such sensations become implanted, as it were, in the cerebral cortex as ' kinaesthetic memories'. These kinaesthetic memories of the motor acts of speech are co-ordinated by a special area situated at the posterior end of the third or inferior frontal convolution of the brain and in the adjacent island of Reil. If this area, known as Broca's area, is destroyed or diseased, expressive, executive or motor aphasia results owing to the loss of kinaesthetic memories for the utterance of words. Similarly, if the development of this area is imperfect or delayed, executive speech is only slowly or imperfectly acquired.

The age at which normal children begin to talk varies considerably. Some children are able to say two or three words at or shortly after the age of twelve months. Many children as a result of imitation will say a few words quite early in their second year, and most normal children can produce short sentences by the age of two years. Admittedly, there are some exceptions when otherwise normal children are later in acquiring adequate speech, even up to the end of their third year. Sometimes such a late development of speech is a family characteristic. In general, however, if a child does not speak by the age of three years, some defect in the mechanism of speech is usually present. It is seldom that a really satisfactory investigation can be carried out at such an early age and the mistake is often made of regarding the child as mentally deficient when, in fact, the speech defect is later shown to be due to an entirely different cause. Usually, therefore, a full investigation has to be postponed until a later age. In practice, one finds that most speech defective children are brought up for examination as they approach school age, that is between four and five years. The first step then is to ascertain whether an absence of intelligible speech or the presence of faulty speech exhibited by a particular child is due to dysarthria, that is, inability properly to articulate, or to a lack of normal function of either the brain as a whole or of one or more of the cortical speech areas. Accurate investigation as to the cause of a child's speech defect is essential, as on the establishment of an exact diagnosis depends not only the prognosis regarding the child's ability to acquire normal or approximately normal speech but also decisions as to the necessary type of treatment and appropriate form of education.

For the complete investigation of speechdefective children at or approaching school age, a co-ordinated team of workers is essential. At
Moor House School for Children suffering from Speech Defects, the team consists of neurologist, psychiatrist, otologist, plastic surgeon, dental surgeon, educational psychologist and speech therapists. In addition, one speech therapist is specially trained in audiometry, including the use of the peep-show audiometer, and facilities exist for consultations with a neuro-surgeon, orthopaedic surgeon and paediatrician, as well as for reference to radiological and electro-encephalographic departments. In many instances, however, it is necessary to admit the child to the in-patient diagnostic section of the School for about a week; out-patient investigation is less satisfactory as affording little or no opportunity for observation and, if the child lives at a distance, several visits to the clinic at short intervals are exhausting for the child and often impracticable for the parents.

\section{Varieties of Speech Disorder in Children}

We find that the children approaching school age referred for investigation fall into one or other of the following seven categories:

(I) Mental deficiency (amentia).

(2) Various forms of congenital or acquired deafness, usually not complete but partial. If a child hears only imperfectly, his reception of spoken language is faulty and he cannot be expected to attain normal speech without accurate diagnosis and appropriate treatment.

(3) Organic disorders of the central nervous system due either to developmental defects, trauma, or to the results of disease. These cases include birth injuries to the brain, perinatal anoxia, kernicterus, and inadequate or faulty development of certain parts of the brain or intracranial nervous structures, that is, various forms of cerebral ' agenesis' (or more strictly ' hypogenesis' which implies under-development) and the effects of bulbar poliomyelitis.

(4) Various forms of congenital, infantile, and juvenile aphasia, both receptive and expressive or executive.

(5) Mechanical defects such as cleft palate, hare-lip, tongue-tie and dental defects.

(6) So-called 'functional' disorders of speech in which there exists no evidence of organic defect or disease. Examples include stammering or stuttering, the dyslalias, various tics and faulty habits of speech, and ' apraxias' of the peripheral organs of speech.

(7) Psychoneurotic, maladjusted and prepsychotic children.

I will now consider each of these categories in more detail, especially from the standpoint of diagnosis.

(I) Mental deficiency. The reference to a depart- 
ment for speech disorders of children in whom absence or delay in the development of speech is due to mental defect is very frequent, and in the investigation of a speech defect, this condition is usually the first that it is necessary to exclude. The diagnosis is made by the application of suitable intelligence tests carried out by an educational psychologist, preferably one with experience in dealing with children suffering from speech disorders. When the child is unable to speak or to understand instructions, the assessment has to be made mainly on performance tests, such as nonverbal items from the Wechsler Intelligence Scale for Children and with appropriate tests from the Stanford-Binet and Collins-Drever scales, in conjunction with observations on the child's general behaviour.

(2) Various forms of deafness. Speech deficiency in children is frequently due to some form of deafness, usually congenital but sometimes acquired in infancy as the result of bilateral otitis media or of meningococcal meningitis, which in some cases involves the auditory nerves, or more rarely, juvenile otosclerosis. If a congenital deafness is complete, the child remains dumb (deaf-mutism) unless taught to lip-read at an early age.

In most children whose speech defect is due to deafness, as seen in a department of speech disorders, the deafness is only partial. These cases are of two varieties, (a) Generalized reduction in auditory acuity, that is, the hearing defect involves the entire range of frequencies, and (b) Highfrequency or high-tone deafness in which the child is deaf only for the higher frequencies; consequently, he cannot hear such sounds as ' $s$ ' and ' sh'. Low-frequency of 'bass' deafness also occurs but is comparatively rare. The loss of hearing in these forms of partial deafness is of the perceptive, inner-ear, or nerve type, and is almost invariably congenital. It is difficult to locate the exact anatomical site of the lesion in these cases but there is some evidence favouring a defect in the auditory area of the cerebral cortex, and in highfrequency deafness, a deficient ' representation' of the higher tones. ${ }^{1}$ Some observers, however, consider that the lesion lies in the cochlea.

The diagnosis of hearing defects in childrenespecially of partial deafness in young childrenis often difficult to establish with certainty, and careful observation and investigation, often over a considerable period, is necessary. A history of rubella in the mother during the earlier months of pregnancy is very significant, for this disease frequently gives rise to various developmental defects in the offspring. The final diagnosis rests on accurate audiometry, repeated at intervals for confirmation. With very young children, however, the use of an ordinary pure-tone audiometer usually impracticable. The 'peep-show' audioe meter, devised by Dr. Hallpike and his co-workers is then most useful to obtain a binaural audiogram? At Moor House School we have found that with. patience and careful training in the application of an ordinary pure-tone audiometer, it is usuall possible to obtain reliable audiograms even if children of five years of age.

When a diagnosis of one of the above types of deafness is established, the case is referred to a appropriate school for the partially deaf for suit ${ }^{\infty}$ able education and training, especially in lip $\vec{\ominus}$ reading.

(3) Organic disorders of the nervous system. Thif్ category comprises a heterogeneous group of cases which include the various forms of cerebrat agenesis or hypogenesis, that is, incomplete otw faulty development, as well as destructive lesion caused by head-injury at birth, perinatal anoxiaoo and by natal and post-natal disease. Among the various forms of cerebral agenesis, we have :-

(a) Congenital cerebral diplegia, also known as 'spastic diplegia' and 'Little's disease', in which the muscles, especially those of the lowe limbs, are spastic and partially paralysed. A feve cases may result from birth injury but mose appear to be due to incomplete development of the motor cells of the Rolandic area and con sequently of the pyramidal tracts. Owing to the deficiency of nerve supply, the muscles of artic lation may also be affected, the result of which is not only delay in the appearance of executive speech but even when some speech is acquired the utterance is jerky, staccato and somewha $\mathbb{2}$ explosive. Further, many cases show varying degrees of mental defect, which is then the primary factor in the delayed appearance of speech

(b) Congenital suprabulbar paresis (which haș also been termed ' congenital pseudo-bulbas paralysis'). In this interesting condition the impaired development is confined to the suprabulbar neurones, that is, the tract of nerve-fibres. proceeding from the motor cortex of the brain too the cranial nerve nuclei of the medulla or bulb ${ }^{3}$ (cortico-bulbar tract) and which supplies the muscles of articulation. The result is varyings degrees of spasticity and paralysis of the lips? tongue, soft palate, laryngeal and pharyngea muscles, either separately or combined. Speech is dysarthric, being slurred and indistinct with deficient lingual and labial sounds.

The extent of the paresis or paralysis varies in? different cases. In what I have termed theo complete syndrome, ${ }^{2}$ the child shows weakness or paralysis of the tongue, even protrusion being ${ }^{\text {? }}$ absent; paresis of the orbicularis oris muscle of the lips with consequent inability to ' round ' the lips $\frac{\rho}{\circ}$ 
also, of the soft palate, together with impairment of swallowing. The mildest manifestation of congenital suprabulbar paresis I believe to be an isolated paralysis or weakness of the soft palate, in association with an increased jaw-jerk. The palatal palsy causes speech to be nasal in character, known as ' rhinolalia'. Similarly, one may meet with an almost isolated paresis of the orbicularis oris or of the tongue. Quite frequently the tongue is protruded fairly well but lateral and upward movement is entirely lacking. Those cases with inability to protrude the tongue are not infrequently mistaken for examples of ' tied tongue', and we have met with several examples in which the frenum linguae has been unnecessarily divided and with no beneficial result.

In severe cases, owing to the weakness of the lips and difficulty in swallowing, the accumulation of saliva is uncontrolled and dribbling is practically continuous. This causes the child considerable anxiety and discomfort, rendering him self-conscious and impairing his social relationships. In the endeavour to relieve this dribbling, in several cases at Moor House School, we have carried out a series of therapeutic experiments, the later ones in conjunction with my colleagues Mr. F. T. Moore and Mr. D. Wynn-Williams of the Plastic Surgical Unit, Queen Victoria Hospital, East Grinstead. Various drugs of the solanaceous series-belladonna and stramonium-which inhibit the secretion of saliva, failed to produce a satisfactory result. Deep X-ray applications to the parotid glands were tried in the hope of reducing glandular activity, but the effect proved only temporary. Next, the duct of each parotid gland (Stensen's duct) was ligatured and in some of the milder cases with satisfactory results. In more severe cases, however, dribbling persisted although in somewhat reduced degree. The earlier of these latter cases seen were relieved by removing one or both submaxillary glands, a procedure which still allows adequate saliva for practical purposes.

In order to improve the rhinolalia (nasal speech) in these cases of suprabulbar paresis, a palatoplasty (Dorrance retro-displacement or so-called 'push back' of the soft palate) or a pharyngoplasty (a palatoplasty combined with a Rosenthal type of pharyngeal flap) has been carried out either by Mr. F. T. Moore or by Mr. D. Wynn-Williams, to assist oro-nasal closure. We found in those cases in which these operations were performed as a 'primary' measure, that is without any excision of salivary glands, both oro-nasal closure and rhinolalia, as well as the power of swallowing, improved, thus increasing the control and disposal of saliva with abolition or reduction in the dribbling. Consequently, either a palatoplasty or a palato-pharyngoplasty is now performed as: a preliminary measure in all cases of isuprabulbar paresis with paralysis or extreme weakness of the palate. If this operation fails to improve salivary control, excision of one or of both submaxillary glands is then carried out.

In children, the diagnosis of congenital suprabulbar paresis has to be made from Parkinsonism resulting from an earlier attack of encephalitis. In these latter cases, however, in addition to a history suggesting encephalitis, the condition does not date from birth and there is usually some muscular hypertonia of the limbs. Also; the spasticity or paresis is not confined to the lips; tongue and palate, but also affects the facial muscles as a whole, with rigidity and poverty of movement. In addition, there is frequently some form of conduct disorder.

(c) Double hemiplegia. In this disorder there is spastic paralysis on both sides of the body including legs, arms, face and tongue. The muscles of articulation, therefore, are affected in the same manner as in congenital suprabulbar paresis but with the addition of spastic weakness or paralysis of all four limbs. In most cases there is a considerable degree of mental deficiency.

(d) Congenital hemiplegia is almost invariably associated with faulty or delayed development of the cortical speech areas resulting in failure of speech to appear at the usual age. The affected cerebral hemisphere, that is, the hemisphere of the brain on the opposite side to the hemiplegia, is under-developed or atrophied, constituting a 'hemicerebral agenesis'. This reduction in size of the affected hemisphere can be well demonstrated by air-encephalography. The exact cause of the hemicerebral deficiency is obscure. Injury to the affected side of the brain at birth may account for a few of the cases but not for the majority, as I have met with the condition in cases of breech-presentation birth, when a headinjury is unusual, and even in children born by Caesarian section. The probable explanation is that of a developmental defect - a hypogenesisthe affected hemisphere failing fully to develop.

If the left hemisphere is affected, the cortical cells subserving motor function as well as those of the potential speech areas fail to develop adequately; consequently the child has a right-sided hemiparesis, is left-handed and the potential speech areas in the right cerebral hemisphere become functional although their development is almost invariably delayed. Nevertheless, even when the hemiplegia is left-sided and the child of normal intelligence, the appearance of executive speech is often retarded.

(e) Bilateral athetosis, also termed ' congenital athetosis' and 'Vogts' syndrome'. Many of the 
children suffering from this condition are of normal intelligence but dysarthria is usually severe. In addition to the involuntary athetoid movements of the limbs and often of the face and tongue, speech is jerky, explosive and indistinct. Many cases can be improved by speech therapy, their speech being rendered at least intelligible.

(f) Ataxias of articulation. In some disorders of the nervous system, articulation is indistinct not from paralysis but from ataxia or inco-ordination of the muscles of articulation. In affections of the cerebellum in children, from injury, relative agenesis of the cerebellum, so-called 'cerebellar encephalitis' and Friedreich's hereditary ataxia, the articulation is slow, laboured and monotonous together with a jerky irregularity. Phonation is sometimes even more affected than articulation and the utterance may be curiously explosive with pronounced separation of the syllables. Occasionally, especially in Friedreich's ataxia, the patient talks as if he has a foreign body in his mouth; this type of speech has been aptly termed ' hot-potato' speech.

In Sydenham's chorea, articulation may be incoordinate owing to sudden jerky movements of the respiratory muscles, tongue and face. Speech, therefore, is hesitant and jerky.

(g) Nuclear and infranuclear affections. These lesions cause dysarthria and include disorders of the bulbar nuclei or of the structures below this level, that is, the peripheral nerves supplying the muscles of articulation and also the muscles themselves.

Facial paralysis occurs in childhood from injury to the facial nerve or from involvement of the nerve by meningitis at the base of the brain, or from spreading suppuration from acute otitis media. Also, the nucleus of the facial nerve may be affected in poliomyelitis. In a special form of myopathy - the facio-scapulo-humeral or Landouzy-Déjèrine type - the muscles of the face are atrophied in addition to those of the upper portion of the arm and shoulder girdle. This affection is usually bilateral and the patient's lower lip may protrude in a characteristic manner which has been termed ' tapir-lip'. In severe facial paralysis, especially if bilateral, the articulation of labial consonants is impaired.

Paralysis of the soft palate, especially from diphtheritic neuropathy and bulbar poliomyelitis, causes difficulty in articulation in the same manner as in congenital cleft-palate, as the naso-pharynx and consequently the nasal cavities cannot be shut off from the mouth. As a result, the voice is nasal, known as 'rhinolalia', and certain consonants are altered (B becomes $M, D$ becomes $N$, and $K$ becomes $\mathrm{Ng}$ ), rendering utterance as a whole very indistinct.
The soft palate may also be paresed as a result of congenital deficiency of its musculature, a diagnosis that can be confirmed only by biopsy. Paralysis of the palate in congenital suprabulbar paresis and double hemiplegia has already been mentioned.

Affections of the hypoglossal nerve-the motor nerve to the tongue-cause paralysis and wasting of the tongue and result from congenital defects of the hypoglossal nucleus (nuclear agenesis), bulbar poliomyelitis and injury to the nerve. If bilateral, the patient will have difficulty in pronouncing linguals.

Lesions of the recurrent laryngeal nerve, usually unilateral, cause abductor paralysis of one vocal cord and render the voice hoarse, and also impair the pronunciation of vowels but do not interfere with the articulation of consonants.

Bulbar poliomyelitis can involve any of the bulbar nuclei, that is of the ninth, tenth, eleventh and twelfth cranial nerves, and cause corresponding paralysis either of the tongue, lips, soft palate, larynx and pharynx, as well as of the trapezius and sternomastoid muscles, either as combined or isolated lesions.

(4) Various forms of aphasia. For practical purposes, cases of aphasia in children can be divided into two main groups :-

(a) Executive or expressive aphasia, in which the child is unable to express himself in spoken language although understanding all that is said to him. He is usually able to indicate his needs by gesture and mime.

(b) Receptive aphasia, in which spoken language is not understood. It follows that in receptive aphasia of congenital origin, the child, being unable to understand what is said to him although he may be otherwise normal, cannot acquire normal speech.

A rare form of congenital receptive aphasia is that of so-called 'word or language deafness', which is probably better termed 'congenital auditory imperception'. Diagnosis is usually difficult but the condition is distinguishable from deafness or partial deafness by the obtaining of a normal audiogram, if and when audiometry is possible. Owing to the disorganization of the speech mechanism which inevitably results from this receptive aphasia, the child often develops a method of vocal expression which is termed 'idioglossia', that is, an individual language consisting of an extreme form of mispronounced and ill-expressed conventional language. When the patient has acquired some speech as a result of treatment, he pronounces his words as he sees them formed by the speaker's lips and as he hears them uttered but he remains unable to distinguish between many word-sounds and his speech is 
somewhat different from that of the normal child, being monotonous and often lacking the usual division into syllables.

In cases we have investigated, it was found that although the outstanding defect was failure to understand the meaning of spoken words, there was, in some examples, an additional inability to appreciate the significance of less specialized sounds such as to distinguish between different types of bell or between different animal noises. In some cases, also, there was a mild but definite degree of actual deafness although quite insufficient to account for the failure to learn the understanding of spoken language. It appeared, therefore, that the designation 'word-deafness' is too limited in scope to apply to this condition; also deafness as such plays no part in most of the cases and very little, if any, in other cases, spoken language being heard but not understood. Consequently, I. M. Allen and I suggested the term ' congenital auditory imperception' as probably describing more completely the fundamental defect. $^{3}$ The pathological basis probably consists in a bilateral agenesis or hypogenesis, that is, incomplete development, of the auditory wordareas in the cortex of the temporal lobes.

Inability to appreciate the significance of symbols such as letters and figures-so-called ' wordblindness', also termed ' alexia ' and 'dyslexia 'is another form of receptive aphasia due to a developmental defect. As compared with the corresponding condition in the auditory fieldcongenital auditory imperception-dyslexia is relatively frequent. Again 'word-blindness' is an unsatisfactory designation in that there is no blindness; the child sees words perfectly but does not understand their meaning. In most cases, the child readily learns to recognize and name single letters and single figures and often words of two letters, but is unable to appreciate the significance of three or more letters and similar groups of figures. With regular and continued individual tuition, many cases gradually improve and eventually acquire some reading ability.

In other examples of aphasia of developmental origin, the aphasia is purely executive or expressive and although the child may be normal in all other respects understanding everything said to him, the appearance of adequate vocal speech is considerably delayed. Such a condition is best termed 'developmental expressive aphasia'. At its first appearance speech is confined to single words, usually reinforced by gesture, but pronunciation is almost invariably faulty, constituting a developmental dysarthria. Provided intelligence is at least average, these cases gradually improve and respond very well to speech therapy.

The failure of speech development in some cases of congenital hemiplegia has already been mentioned. This deficiency, also, is of the nature of an executive or expressive aphasia.

Acquired expressive or executive aphasia is also met with in children of school age as a result of a lesion of the inferior left frontal convolution in right-handed children and vice-versa in lefthanded children. Such a lesion can follow head injury, brain-abscess and cerebral tumour, and occasionally encephalitis complicating one or other of the exanthemata. In cases of traumatic executive aphasia, the response to speech therapy is usually very good, but when the aphasia follows cerebral abscess the results are not so satisfactory and in addition to other factors, the child frequently suffers from epilepsy. Tumours involving the speech area that are amenable to total removal are very rare, but in the few examples met with - meningioma and oligodendroglioma - the results of speech therapy have been very good.

Total aphasia, that is a combination of both receptive and expressive aphasia, may follow bilateral head injuries and some forms of encephalitis, especially measles encephalitis. In these cases, the child loses both the understanding of spoken language and the faculty of executive speech. Treatment is usually a slow and laborious process, but in several cases, the ultimate results have been well worth the trouble and time expended.

(5) Mechanical defects. These include such conditions as congenital cleft-palate, with or without hare-lip ; a congenitally short soft palate; a relatively immobile tongue owing to a short frenum; and, in older children, various dental defects. The child with a cleft palate exhibits a characteristic nasal speech known as 'rhinolalia'. Provided closure of the naso-pharynx by a mobile soft palate has been secured by suitable surgical measures, the response to speech therapy in most cases is excellent.

Tongue-tie is relieved by incision of the shortened frenum. As I have previously said, several of our cases of congenital suprabulbar paresis, elsewhere and at an earlier age had been mistaken for tongue-tied children owing to the relative immobility of the tongue and were subjected to an un necessary operation.

(6) Functional disturbances. These comprise disorders of speech with no recognizable organic or structural defect either in the nervous system or of the peripheral organs of articulation, and include cases of stammering or stuttering and the various forms of dyslalia.

Stammering and stuttering are terms used synonymously to denote a spasmodic type of speech disorder with which we are all very familiar. Strictly speaking 'stammer' represents 
the hesitant utterance and 'stutter' the element of repetition in the defect but the distinction is of no practical importance. In Great Britain, the term 'stammering' is more widely used to describe this type of speech disorder, while 'stuttering' seems to be preferred in America.

Physiological stammering, sometimes termed ' primary stuttering' consists in hesitation, repetitions and prolongations in speech which often occur during the normal development of language between the ages of two and four years as purely transient phenomena. Such disturbances of speech are unaccompanied by awareness or anxiety.

Pathological stammering or ' secondary stuttering' are terms applied to the more persistent stammer which usually appears between four and seven years of age, and in some cases occurs as a prolongation of a stage of physiological or primary stammer.

There has for long been considerable controversy concerning the causation of stammering and several theories have been propounded in the attempt to explain the phenomenon. The subject has been complicated by the fact that many observers who have studied the condition have done so only in adolescents and adults. By this time, possible causative factors have become obscured by many secondary manifestations, mainly psychological, such as self-consciousness, fear of ridicule, and feelings of inferiority. The observer of these effects has had the inevitable tendency to find psychopathological explanations for the primary speech defect, as well as for the secondary manifestations.

In the majority of cases, the child starts to talk quite normally, but begins to stammer between the ages of four and seven years. Stammer is much more common in boys than in girls-fully 80 to 90 per cent. of the cases are boys. Lefthanded tendencies have been present in 60 per cent. of cases and can nearly always be traced in parents or near relatives. By the time the stammer is established, the boy is right-handed, at least for the purpose of writing. When seen at the onset of stammer, the children are usually quite normal in all other respects, and there are seldom any grounds for describing them as more 'nervous', that is psychologically disturbed or maladjusted, than other children.

Among the hypotheses that have been advanced in explanation of the causation and mechanism of stammering are :-

(a) The theory of ' handedness', that is, the attempt to convert an inherently left-handed child into a right-handed one. Such outside interference with the development of a dominant cerebral hemisphere in integrating the mechanism neces- sary for normal speech may lead to a stammer. From the age of two the child begins to establish his handedness, and also his habits of speech. In most individuals the left cerebral hemisphere becomes dominant, and consequently the higher speech areas are located on the left side of the brain in association with right-handedness. Some delay in the onset of speech and in the preferential use of either hand is not uncommon in the history of children who begin to stammer at the early age of three years. A second critical period is when the child is just beginning to learn to read and write, that is, to acquire graphic language, and is attempting to correlate these two new unilateral cerebral functions with speech, which itself is still in a somewhat developmental stage. Thus, if an inherently left-handed child is thwarted in the dominant use of his left hand at the time his education begins, he is liable to develop a stammer. The reverse also applies, that is, the attempt to convert an innate right-handed child to the dominant use of the left hand. H. Gordon ${ }^{4}$ describes an endeavour to train children in a special school to use the left hand, the result being a wholesale development of stammer.

(b) The theory of deficient auditory imagery of words as the primary defect, that is, defective word-memory in the sense of an inability to retain mental pictures of words and sentences.

(c) The theory of asymmetry in functionlack of harmony between the three sets of muscle systems necessary for speech, viz., respiratory, vocal and articulatory, in association with emotional disturbances such as anxiety, apprehension and embarrassment. These two factors-physical and psychological - thus constitute a vicious circle.

(d) The theory that stammering is purely psychological in origin and dependent on emotional disturbance and maladjustment. The psychoanalytical view, based on the observations of Freud, postulates that stammering results from the attempt to repress from consciousness, into the unconscious, various painful memories and disturbing thoughts in order that they may not be betrayed by speech.

Although ' handedness' undoubtedly plays some part in the aetiology of stammering, it is probable that the disorder has a multiple origin which time and space do not allow of discussion in this communication. Also, it must be admitted that the treatment of stammering is, as yet, by no means satisfactory.

Many stammerers acquire various tricks, chiefly as a result of their efforts to overcome or mask the stammer. Thus, extra noises may be thrown in, for example, sudden inspiratory gruntings or whooping noises, or the patient may make facial grimaces and even contortions of his limbs. 
Various articulative tics or habit-spasms are met with in nervous and psychopathic children, sometimes in conjunction with some degree of stammer. The child's speech may be interrupted by a weird pharyngeal barking or grunting noises. Articulation may be monosyllabic, an extra breath being taken for each syllable. Again, speech may be jumbled up in the most extraordinary manner, although the 'tiqueur' usually interpolates, now and again, a clearly articulated sentence among other unintelligible phrases.

A purely hysterical stammer is uncommon in children. When present, it is more frequent in girls than in boys and usually appears at about the time of puberty or later. Some cases of hysterical stammer result from mimesis, that is, attempted imitation of a true stammerer; as a rule the children are somewhat maladjusted. Appropriate treatment usually yields satisfactory results. Similarly ' hysterical mutism', so common as a manifestation of a war psychoneurosis, is rare in young children but it is occasionally met with in older girls as a result of emotional disturbance and auto-suggestion.

The condition known as 'lalling' is a normal phase in the development of speech in childhood. The form of words is changed by substituting one sound for another, or by the omission and ellision of some of the syllables. Also, there may be a lack of precision in the pronunciation of certain consonants, for example, the substitution of the uvular $\mathbf{R}$ for the ordinary laryngo-palatal $\mathbf{R}$, or $\mathrm{V}$ for $\mathrm{Th}, \mathrm{W}$ for $\mathrm{R}$, so that ' broken reed ' becomes ' bwoken weed'. When lalling persists beyond the usual age for adjustment, the condition is termed 'dyslalia'. Several varieties of dyslalia are recognised, the most important of which are as follows :- 'Simple dyslalia' consists in the defective articulation of one consonant or one pair of consonants, e.g. ' edephant' or ' evephant' for elephant and 'ting' for king. When several sounds are mispronounced, the term 'multiple dyslalia' is used, while ' general dysilalia' implies the defective articulation of numerous sounds and the mutilation of many words, together with deficiencies of vocabulary and syntax. 'Lisping', also termed 'sigmatism ', consists in the substitution of $\mathrm{Th}$ for $\mathrm{S}$.

In exceptional cases of general dyslalia, although most of the vowels are pronounced correctly, the consonants are so mutilated, mispronounced and substituted that the child appears to be speaking a language entirely of his own, constituting a form of 'idioglossia'. Although unintelligible to those not acquainted with the child, his language is usually well understood by parents and others continually in contact with him.

With the gradual acquisition of speech in cases of developmental expressive aphasia, such speech is frequently dyslalic until corrected by appropriate therapy.

In some cases, a dysarthria is due to an apparent ' apraxia' of the lips, tongue or soft palate, either separately or in combination, that is, faulty or inefficient use of these peripheral organs of speech in the absence of any physical signs of disorder of organic origin. Usually, the condition gradually improves, especially with speech therapy.

(7) Psychoneurotic and pre-psychotic children. In these cases, there is considerable maladjustment to ordinary surroundings and the speech defect is usually one of retarded and inadequate speech development due to purely psychological causes. Speech, when it appears, almost invariably assumes the form of a severe dyslalia.

\section{REFERENCES}

1. WOOLSEY, C. N., and WALZ, E. M. (1942), Bull. Fohns Hopk. Hosp., $121,135$.

2. WORSTER-DROUGHT, C. (1956), F. Laryng., 70, 453. 3. WORSTER-DROUGHT, C., and ALLEN, I. M. (1929),
f. Neurol. Psychopath., 9, 193 and (1930), 10, 193; also

WORSTER DROUGHT, C. (1943), Med. Press, 210, 411. 4. HOBHOUSE, N. (quoted by) (1932), 'Nervous Disorders in
Infancy and Childhood', London, p. 140.

\section{RUTHIN CASTLE, NORTH WALES}

A Clinic for the diagnosis and treatment of Internal Diseases (except Mental or Infectious Diseases). The Clinic is provided with a staff of doctors, technicians and nurses.

The surroundings are beautiful. The climate is mild. There is central heating throughout. The annual rainfall is $\mathbf{3 0 . 5}$ inches, that is less than the average for England.

The Fees are inclusive and vary according to the room occupied.

- For particulars apply to THE SECRETARY, Ruthin Castle, North Wales.

Telegrams: Castle, Ruthin

Telephone: Ruthin 66 